\title{
Nutritional and feeding management Studies on hybrid Nile tilapia (Oreochromis niloticus $x$ O. aureus)
}

\section{Ahmed K. I. El-Hammady; Elham A.Wassef ; Mohammed I.Wafa and Raky F.Attalla.}

Department of Fish Nutrition, National Institute of Oceanography and Fisheries; Kasr El-Aini Street, Cairo, Egypt

Email: akelhammady@yahoo.com.

\begin{abstract}
$\mathrm{T}$ wo experiments were performed in two or three way factorial design on Nile tilapia hybrid (O. niloticus $x$ O. aureus) at the El kanater El khairyia Experimental Fish Research Station belonging to NIOF. The three experiments were carried out in outdoor concrete tanks, each of $10 \mathrm{~m}^{3}$ total water volume. Both experiments lasted 20 weeks after start and fish initial weights for the first and second experiments were 30.24 and $31.76 \mathrm{~g}$, respectively.

The first trial aimed to test the interaction between protein ( 25 or $30 \%$ p25 or p30) and dietary energy levels (15.70; 16.74 or $18.84 \mathrm{Kj}$ g-1 dry diet) on growth performance of hybrid tilapia. In the second experiment, three feeding rates $(2,4$ or $6 \%$ biomass of fish $\mathrm{R} 2$;R4 or R6) within each rate two feeding frequencies (2 or 3 times /day F2 or F3 ) within each two stocking densities (25 or 50 fish $/ \mathrm{m}^{3} \mathrm{D} 25$ or D50) were tested for their effects on growth performance of hybrid tilapia. Results of the first experiment revealed that the maximum growth performance of hybrid tilapia was obtained using the diet P25\% E16.74KJ, which is recommended for hybrid tilapia.

Results of the second trial recommended the feeding rate; frequency and stocking density of $4 \%$; two times feeding at $25 \mathrm{fish} / \mathrm{m}^{\mathrm{i}}$ density, respectively for best growth performance of hybrid tilapia.
\end{abstract}

Key words: hybrid tilapia, growth energy, protein, feeding ratio, feeding frequency, stocking denity.

\section{INTRODUCTION}

Nutrition of tilapias is an area of research that has received great attention, because of the influence of nutrition on fish growth and health as well as on the cost of production. Immense literature on tilapia culture and feeding is available and has been more recently reviewed by El-Sayed (2006).

The optimal P/E ratio may vary among fish species, fish size, diet formulation and culture system (Ai et al., 2004). Winfree \& Stickney (1981) revealed that the optimum P/E ratio for Oreochromis aureus fry was 125 (56\% protein and gross energy $4600 \mathrm{~K} \mathrm{cal} / \mathrm{Kg}$ diet), however for the same fish $7.5 \mathrm{~g}$, the optimum P/E ratio was 108 (34\% protein and gross energy $3200 \mathrm{~K} \mathrm{cal} \mathrm{/} \mathrm{Kg}$ 
diet). So, to obtain optimum growth for Oreochromis niloticus fry (2.9 g) $75 \mathrm{mg}$ $\mathrm{P} / \mathrm{K}$ cal digestible energy are required (Santiago \& Reyes, 1993).

Furthermore, in finfish aquaculure, stocking density can not only affect fish growth, but has also been shown to alter behavioral interactions (Baskerville -Bridges \& Kling, 2002). Therefore, determining the effects of stocking density in different fish species is vitally important in commercial culture (Woods, 2003).

NRC (1993) showed that the best feeding frequencies recorded for various sizes of tilapia which ranged between two day old to (1-5g), 5-20 g , 20$100 \mathrm{~g}$ and $>100 \mathrm{~g}$ reared at $28{ }^{\circ} \mathrm{C}$ as $8,6,4$ and three times per day, using feeding levels of $30-10 \%, 10-6 \%, 6-4 \%$ and $3 \%$ of fish body weight, respectively.

Therefore, the present work was carried out to determine the optimum dietary protein level with gross energy levels and feeding frequency for hybrid tilapia (O. niloticus $X O$. aureus) and to investigate the interaction between stocking density and feeding regime of hybrid tilapia when raised in concrete ponds.

\section{MATERIALS AND METHODS}

Feeding trials were conducted at El-Kanater El-khayria Experimental Fish Farm (EEF), National Institute of Oceanography and Fisheries (NIOF) Egypt. Twelve $10 \mathrm{~m}^{3}$ concrete tanks, with natural water supply-through water system from the adjacent River Nile branch, were used for each trial.

\section{Experimental Fish}

Hybrid tilapia (female $O$. niloticus $X$ male $O$. aureus) juveniles were obtained (from a private hatchery) and maintained in the experimental concrete tanks at a stocking rate of $25 \mathrm{fish} / \mathrm{m}^{3}$ for 3 weeks for an acclimatization period before the commencement of first growth trial. During this period, fish were fed on a commercial tilapia feed ( $30 \% \mathrm{CP}$ ) for 10 days then switched to the experimental diets.

At start of feeding experiments $1 \& 2$, fish of each concrete tank were collectively weighed and average initial weights per treatment (IW, g/fish) were 30.24 and 31.76 g respectively.

\section{Experimental design}

In feeding trial 1, three dietary gross energy levels (E1, 15.70; E2, 16.74; E3 $18.84 \mathrm{Kj} \mathrm{g}^{-1}$ dry diet) within each two protein (P) levels (25 or 30\%) were tested (Table 1) as duplicates for each treatment. Fish were fed on the test diets dry pellets at a rate of $3 \%$ of biomass twice per day, six days a week for 20 weeks at ambient temperature ranged between 23 and $25^{\circ} \mathrm{C}$. Dissolved oxygen (DO) ranged between $4.8 \& 6.3 \mathrm{mg} / \mathrm{L}, \mathrm{pH}, 6.9-7.9$ and ammonia, 0.23-0.29 mg/L (APHA, 1995). 
Table 1. Ingredients and proximate composition (\% $D M$ ) of test diets fed to hybrid tilapia (O. niloticus XO. Aureus) in trial 1.

\begin{tabular}{|c|c|c|c|c|c|c|}
\hline \multirow{2}{*}{$\begin{array}{c}\text { Gross energy } \\
\text { (Kjg }^{-1} \text { dry diet)* } \\
\text { Dietary protein } \\
(\%)\end{array}$} & \multicolumn{2}{|c|}{15.70} & \multicolumn{2}{|c|}{16.74} & \multicolumn{2}{|c|}{18.84} \\
\hline & 25 & 30 & 25 & 30 & 25 & 30 \\
\hline \multicolumn{7}{|l|}{ Ingredients } \\
\hline Fish meal (herring) & 15 & 18 & 18 & 23 & 22 & 27 \\
\hline Soybean meal $^{1}$ & 4 & 15 & 8 & 14 & 7 & 10 \\
\hline Yellow corn flour & - & - & 34 & 30 & 45 & 30 \\
\hline Wheat bran & 77.5 & 64.0 & 34 & 29.5 & 13.5 & 21.5 \\
\hline Sunflower oil (SO) & 1.5 & 1.0 & 4 & 1.5 & 10.5 & 9.5 \\
\hline Vitamin \& & 2 & 2 & 2 & 2 & 2 & 2 \\
\hline $\begin{array}{l}\text { Minerals mix } \\
\text { Composition } \\
\text { (\%DM)Total }\end{array}$ & 100 & 100 & 100 & 100 & 100 & 100 \\
\hline Moisture & 10.13 & 10.09 & 10.06 & 10.09 & 9.35 & 9.13 \\
\hline Crude protein, (P) & 25.27 & 30.22 & 24.95 & 30.11 & 24.96 & 29.91 \\
\hline Ether extract, (EE) & 5.90 & 5.25 & 8.17 & 5.82 & 14.57 & 13.80 \\
\hline Crude fiber, (CF) & 7.90 & 7.05 & 4.53 & 4.22 & 2.74 & 3.30 \\
\hline $\mathrm{NFE}^{3}$ (by & 42.91 & 40.70 & 45.72 & 42.61 & 42.39 & 36.92 \\
\hline difference) & 7.89 & 6.69 & 6.57 & 7.15 & 5.99 & 6.94 \\
\hline Ash & 15.68 & 15.77 & 16.99 & 16.74 & 18.94 & 18.87 \\
\hline \multicolumn{6}{|l|}{$\mathrm{P} / \mathrm{E}$ ratio } & 158.51 \\
\hline $\begin{array}{cl}1- & \text { Solvent extr } \\
2- & \text { Each } 1 \mathrm{~kg} \text { of } \\
& 0.8 \mathrm{~g} \text { vit K, } 4 \\
& \text { Folic acid, } 2 \\
& \text { g Manganes }\end{array}$ & $\begin{array}{l}\text { itamins a } \\
\text { g vit } B_{2} \text {, } \\
\text { mg Bioti } \\
\text { 22g Zinc }\end{array}$ & $\begin{array}{l}\text { minerals I } \\
200 \text { git. } \mathrm{B}_{6} \text {, } \\
.04 \mathrm{~g} \text { Selen }\end{array}$ & $\begin{array}{l}\text { xture conta } \\
\text { g pantother } \\
\text { e chloride, } \\
\text { m. }\end{array}$ & $\begin{array}{l}\text { ed: } 4.8 \mathrm{~m} .1 . \\
\text { acid. } 8 . \mathrm{g} \\
\text { g Copper, } 0\end{array}$ & $\begin{array}{l}\text { vit. } \mathrm{D}_{3}, 4.0 \\
\text { icotinic aci } \\
\text { g Iodine, } 1\end{array}$ & $\begin{array}{l}\text { vit. E, } \\
400 \text { mg } \\
\text { g Iron, } 22\end{array}$ \\
\hline $\begin{array}{l}\text { 3- Nitrogen fre } \\
\text { 4- } \quad * \mathrm{Kj}=4.186 \mathrm{~K}\end{array}$ & xtract. & & & & & \\
\hline
\end{tabular}

In trial 2, three feeding rates (2, 4 and $6 \%$ of wet weight/day), dry diet at two frequencies (0900 and $1500 \mathrm{~h})$ or $3(0900,1200,1500 \mathrm{~h})$ feedings/d, $\mathrm{F}_{2}, \mathrm{~F}_{3}$ ) with each feeding rate was offered to duplicate groups of young hybrid tilapia stocked in concrete tanks at two densities (25 or $50 \mathrm{fish} / \mathrm{m}^{3}, \mathrm{D}_{25}, \mathrm{D}_{50}$ ) for 20 weeks with provided a single diet (25\% CP, $16.74 \mathrm{Kj} \mathrm{g}^{-1}$ dry diet, (Table 1). Ambient temperature ranged from $22.3-25.6^{\circ} \mathrm{C}$; DO, 5-6 mg/L; $\mathrm{pH}, 6.9-7.7$ and ammonia, 0.23-0.29 mg/L.

For both trials, fish growth was monitored biweekly (14d) and feed ration rate of each treatment was then readjusted accordingly.

\section{Analytical procedures}

Samples of 15 fish were taken at start and end, from each experiment, for the initial and final proximate flesh analyses respectively. Feed ingredients and experimental diets were analyzed for moisture (drying at $80-85^{\circ} \mathrm{C}$ to 
constant weight), and lipid content of flesh by Bligh and Dyer (1959) crude protein (semi-automatic Kjeldahl, VELP Scientifica UDK 126), lipids (Soxhelt using a Petrolume ether a solvent), crude fiber and ash content (ignition in a muffle furnace at $550^{\circ} \mathrm{C}$ for $6 \mathrm{~h}$ ) adopting the standard methodology of AOAC (1995).

\section{Data and statistical analyses}

Gross energy (GE) in diet (Kcal) $(1 \mathrm{Kj}=4.186 \mathrm{Kcal})$ was calculated according to NRC (1993) calculated $=5.64 \mathrm{X} \% \mathrm{CP}+9.44 \mathrm{X} \% \mathrm{EE}+4.11 \mathrm{X} \%$ NFE. The following growth and feed utilization indices were used for statistical analysis: total weight gain (WG, g/fish) = final body weight (FW) - initial body weight (IW); specific growth rate $(\mathrm{SGR}, \% / \mathrm{d})=100(\ln \mathrm{FW}-\ln \mathrm{IW}) / \mathrm{t}$ (experimental period in days).

Measurements calculatied and determined are 1- Apparent food conversion ratio (FCR) (because we did not measure the uneaten food) = amount of dry feed supply (g)/body weight gain of fish (g).

2- apparent protein efficiency ratio $(\mathrm{PER})$ = fish weight gain (g)/ amount of protein fed (g).

3- apparent protein productive value $(\mathrm{PPV} \%)=$ body protein gain / dietary protein intake.

4- energy retained $(E R \%)=($ body energy gain / dietary energy intake $) \times 100$.

Data were subjected to ANOVA statistical analyses using a software SAS (1996) version 6.12 (SAS Inst., Cary, NC 27513, USA). Means of different parameters were compared by Duncan's multiple range test at significance level $P<0.05$.

\section{RESULTS}

\section{A. Growth and feed utilization Indices}

The measured growth indices (WG, g/fish; SGR, \%/d) as well as feed utilization indices (FCR, PER, PPV) for the six treatments of feeding trial 1 are presented in (Table 2).

Table 2. Growth performance and feed utilization indices of hybrid tilapia (O. niloticus $X O$. aureus) in trial 1 (mean $\pm \mathrm{SE}$ ).

\begin{tabular}{|c|c|c|c|c|c|c|c|}
\hline Treatment & $\begin{array}{c}\text { WG } \\
\text { (g/fish) }\end{array}$ & $\begin{array}{c}\text { SGR } \\
(\% / d)\end{array}$ & $\begin{array}{c}\text { Feed } \\
\text { supply (g) }\end{array}$ & FCR & PER & PPV (\%) & ER (\%) \\
\hline \multicolumn{8}{|c|}{ Gross Energy (Kjg ${ }^{-1}$ dry diet) } \\
\hline E1 (15.70) & $108.40^{\mathrm{b}} \pm .2$ & $1.19^{\mathrm{b}} \pm .02$ & 302.88 & $2.80^{\mathrm{ab}} \pm .05$ & $1.30^{\mathrm{ab}} \pm .02$ & $19.96^{\mathrm{b}} \pm 1.41$ & $12.80^{\mathrm{a}} \pm 1.26$ \\
\hline E2 (16.74) & $116.12^{\mathrm{a}} \pm .1$ & $1.13^{\mathrm{a}} \pm .03$ & 312.24 & $2.69^{\mathrm{b}} \pm .02$ & $1.40^{\mathrm{a}} \pm .01$ & $21.53^{\mathrm{a}}+1.04$ & $12.37^{\mathrm{ab}} \pm 1.11$ \\
\hline E3 (18.84) & $094.42^{\mathrm{c}}+.1$ & $1.02^{\mathrm{c}}+.02$ & 277.58 & $2.94^{\mathrm{a}}+.04$ & $1.25^{\mathrm{b}}+.04$ & $19.86^{\mathrm{b}}+1.29$ & $11.29^{\mathrm{b}}+1.07$ \\
\hline \multicolumn{8}{|c|}{ Dietary protein $(\mathrm{P})$} \\
\hline $25 \%(\mathrm{P} 25)$ & $105.54^{\mathrm{b}} \pm 1$ & $1.07^{\mathrm{a}} \pm .04$ & 359.36 & $2.82^{\mathrm{a}} \pm .03$ & $1.44^{\mathrm{a}} \pm .03$ & $22.50^{\mathrm{a}} \pm 1.10$ & $12.35^{\mathrm{a}} \pm 1.04$ \\
\hline $30 \%($ P30) & $107.08^{\mathrm{a}} \pm 1$ & $1.08^{\mathrm{a}}+.03$ & 365.67 & $2.80^{\mathrm{a}} \pm .05$ & $1.19^{\mathrm{b}}+.04$ & $18.40^{\mathrm{b}}+1.25$ & $11.95^{\mathrm{a}} \pm 1.12$ \\
\hline \multicolumn{8}{|l|}{$\mathbf{E} \mathbf{X} \mathbf{P}$} \\
\hline E1 x P25 & $099.38^{\mathrm{bc}} \pm 1$ & $1.04^{\mathrm{b}} \pm .01$ & 285.22 & $2.87^{\mathrm{a}} \pm .05$ & $1.38^{\mathrm{b}} \pm .03$ & $21.07^{\mathrm{b}} \pm 1.72$ & $12.32^{b} \pm 1.03$ \\
\hline E1 x P30 & $117.41^{\mathrm{a}-1}$ & $1.13^{\mathrm{a}+.08}$ & 320.53 & $2.73^{\mathrm{b}} \pm .04$ & $1.21^{\mathrm{c}} \pm .05$ & $18.84^{\mathrm{c}} \pm 1.13$ & $13.28^{\mathrm{a}} \pm 1.11$ \\
\hline E2 x P25 & $119.97^{\mathrm{a}} \pm 2$ & $1.14^{\mathrm{a}} \pm .03$ & 319.12 & $2.66^{\mathrm{c}} \pm .05$ & $1.57^{\mathrm{a}} \pm .06$ & $24.44^{\mathrm{a}} \pm 1.21$ & $12.60^{\mathrm{b}} \pm 1.54$ \\
\hline E2 x P30 & $112.26^{\mathrm{b}} \pm 1$ & $1.11^{\mathrm{a}} \pm .07$ & 305.35 & $2.72^{\mathrm{b}} \pm .06$ & $1.22^{\mathrm{c}} \pm .04$ & $18.62^{\mathrm{c}} \pm 1.37$ & $12.13^{\mathrm{b}} \pm 1.29$ \\
\hline E3 x P25 & $097.26^{c}+2$ & $1.03^{\mathrm{b}} \pm .02$ & 285.94 & $2.94^{\mathrm{a}}+.04$ & $1.36^{\mathrm{b}}+.04$ & $21.99^{\mathrm{b}} \pm 1.61$ & $12.14^{\mathrm{b}} \pm 1.31$ \\
\hline E3 x P30 & $091.57^{\mathrm{d}} \pm 1$ & $1.00^{\mathrm{b}}+.03$ & 269.22 & $2.94^{\mathrm{a}} \pm .05$ & $1.14^{\mathrm{d}+.03}$ & $17.73^{\mathrm{d}} \pm 1.23$ & $10.44^{\mathrm{c}}+1.12$ \\
\hline
\end{tabular}

a,b,c and $\mathrm{d}$ values in the same row bearingdifferen superscript letters are significantly different $(P<0.05)$. 
Results of trial 1 revealed that increasing dietary protein level from 25 to $30 \%$ had slightly improved weight gain, (WG), specific growth rate, SGR and feed conversion ratio, FCR. However, increasing protein level significantly reduced $(\mathrm{P}<0.05)$ both protein efficiency ratio, $(\mathrm{PER})$ and protein productive value, (PPV) (Table 2).

In the mean time, optimal gross energy which produced significantly $(P<0.05)$ highest growth among all treatments was $16.74 \mathrm{Kj} \mathrm{g}^{-1}$ dry diet regardless of protein level. Further increase in gross energy up to $18.84 \mathrm{Kj} \mathrm{g}^{-1}$ dry diet had negatively affected growth (WG, SGR) and nutritional indices (FCR, PER, PPV and ER) (Table 2). As for the interaction between dietary protein level, and gross energy, results indicated that the 25\% CP diet with 16.74 $\mathrm{Kj} \mathrm{g}^{-1}$ dry diet had yielded a the best relative growth as well as the feed utilization among all the tested diets.

Results of trial 2 show that the lowest tested feeding rate (2\% of biomass, $\mathrm{R}_{2}$ ) showed significantly inferior growth (WG, SGR), whereas higher feed rates ( 4 and $6 \%$ of biomass, $\mathrm{R}_{4} \& \mathrm{R}_{6}$ ) had significantly $(P<0.05)$ improved all growth indices (Table 4). Therefore, it is more economical to adopt the $4 \%$ biomass feeding rate as the daily feed ration for the best fish growth performance. On the other hand, both tested feeding frequencies (two, $\mathrm{F}_{2} / \mathrm{d}$, or three feedings, $\left.F_{3} / d\right)$ did not exhibit significant impact $(P>0.05)$ over growth or feed utilization indices (Table 4). However, increasing stocking density from $D_{25}$ to $D_{50} / \mathrm{m}^{3}$ decreased growth rate for fish. Considering the interaction between mixed feeding schedules adopted in trial 2 and based on the measured indices, treatment $\mathrm{R}_{4} \mathrm{~F}_{2} \mathrm{D}_{25}$ led to the best growth (Table 4) for young hybrid tilapia (O. niloticus X O. aureus) reared in concrete tanks.

\section{B. Fish body composition}

In Trial (1) results of the effect of using different dietary protein levels (25 or 30\%), gross energy (15.70-18.84 Kj g ${ }^{-1}$ dry diet) on proximate analysis of flesh are shown in Table (3). Data indicated that fish fed both protein level (25 or $30 \%$ ) did not record significant differences on body chemical composition $(\mathrm{P}>0.05)$. Varied gross energy ratios had significantly $(\mathrm{p}<0.05)$ affected both lipid and ash contents of fish but not moisture and protein. 
Ahmed K. I. El-Hammady et al.

Table 3. Proximate analyses (\%wet weight) of hybrid tilapia muscles fed test diets in trial $1 . .($ mean $\pm \mathrm{SE})$

\begin{tabular}{|c|c|c|c|c|c|}
\hline Treatment & Moisture & Crude protein & Crude lipid & Ash & Gross energy $^{*}\left(\mathrm{Kjg}^{-1}\right)$ \\
\hline Initial & $7619 \pm 1.21$ & $15.72+1.05$ & $3.92+0.9$ & $4.17 \pm 0.40$ & $5.31 \pm 0.12$ \\
\hline \multicolumn{6}{|c|}{ Gross Energy (k cal/kg dry diet) } \\
\hline${ }_{1} \mathrm{E}(15.70)$ & $76.57 \pm 2.18^{\mathrm{a}}$ & $15.39+1.24^{\mathrm{a}}$ & $4.75+0.80^{b}$ & $3.27 \pm 0.82^{b}$ & $5.56^{\mathrm{b}} \pm 0.18$ \\
\hline${ }_{2} \mathrm{E}(16.74)$ & $76.35+2.08^{\mathrm{a}}$ & $15.40 \pm 1.56^{\mathrm{a}}$ & $4.71 \pm 0.80^{\mathrm{b}}$ & $3.51 \pm 0.73^{\mathrm{ab}}$ & $5.55^{\mathrm{b}}+0.15$ \\
\hline${ }_{3} \mathrm{E}(18.84)$ & $75.62 \pm 2.34^{\mathrm{a}}$ & $15.71 \pm 2.04^{\mathrm{a}}$ & $5.10 \pm 0.70^{\mathrm{a}}$ & $3.58+0.86^{\mathrm{a}}$ & $5.79^{\mathrm{a}}+0.17$ \\
\hline \multicolumn{6}{|c|}{ Dietary protein (P) } \\
\hline CP 25\% & $75.97 \pm 2.70^{\mathrm{a}}$ & $15.58+1.85^{\mathrm{a}}$ & $4.89 \pm 0.57^{\mathrm{a}}$ & $3.54 \pm 0.92^{\mathrm{a}}$ & $5.66^{\mathrm{a}}+0.20$ \\
\hline CP $30 \%$ & $76.38+2.03^{\mathrm{a}}$ & $15.42 \pm 1.02^{\mathrm{a}}$ & $4.81 \pm 0.77^{\mathrm{a}}$ & $3.36+0.76^{\mathrm{a}}$ & $5.59^{\mathrm{a}}+0.15$ \\
\hline \multicolumn{6}{|l|}{$\mathbf{E} \times \mathbf{P}$} \\
\hline E1 x P25 & $76.84+3.24^{\mathrm{a}}$ & $15.28+1.56^{\mathrm{a}}$ & $4.63+0.47^{b}$ & $3.22 \pm 0.95^{b}$ & $5.52^{\mathrm{C}}+0.16$ \\
\hline E1 x P30 & $76.29+2.54^{\mathrm{a}}$ & $15.49+2.54^{\mathrm{a}}$ & $4.87+0.57^{\mathrm{ab}}$ & $3.32+0.73^{\mathrm{b}}$ & $5.63^{b}+0.11$ \\
\hline E2 x P25 & $75.97 \pm 2.29^{\mathrm{a}}$ & $15.54+1.92^{\mathrm{a}}$ & $4.80 \pm 0.82^{\mathrm{ab}}$ & $3.66+0.65^{\mathrm{a}}$ & $5.62^{\mathrm{b}} \pm 0.13$ \\
\hline E2 x P30 & $76.73+2.25^{\mathrm{a}}$ & $15.26 \pm 2.54^{\mathrm{b}}$ & $4.62+0.95^{\mathrm{b}}$ & $3.35 \pm 0.93^{\mathrm{b}}$ & $5.48^{\mathrm{c}}+0.12$ \\
\hline E3 x P25 & $75.11+3.11^{\mathrm{a}}$ & $15.91+1.78^{\mathrm{bc}}$ & $5.25+0.94^{\mathrm{a}}$ & $3.75+0.83^{\mathrm{a}}$ & $5.89^{\mathrm{a}}+0.14$ \\
\hline E3 x P30 & $76.13+1.25^{\mathrm{a}}$ & $15.51 \pm 2.04^{\mathrm{bc}}$ & $4.95+0.27^{\mathrm{a}}$ & $3.40 \pm 0.65^{\mathrm{ab}}$ & $5.67^{\mathrm{b}}+0.13$ \\
\hline
\end{tabular}

a,b,c and $d$ values in the same row with different superscript letters are significantly different $(P<0.05)$.

* Muscle gross energy (K cal/100g muscle) by NRC (1983) $=5.7 \mathrm{x} \%$ crude protein + 9.5 x \% crude lipid. $(\mathrm{Kj}=4.186 \mathrm{~K} \mathrm{cal})$

Table 4. Growth performance and feed utilization indices of hybrid tilapia fed test diets in trial 2 (mean $\pm \mathrm{SE}$ ).

\begin{tabular}{|c|c|c|c|c|c|c|c|}
\hline Treatment & $\begin{array}{c}\text { WG } \\
\text { (g/fish) }\end{array}$ & $\begin{array}{l}\text { SGR } \\
\text { (\%/d) }\end{array}$ & $\begin{array}{c}\text { Feed } \\
\text { supply (g) }\end{array}$ & FCR & PER & PPV (\%) & ER (\%) \\
\hline \multicolumn{8}{|l|}{ Feed ration $(R)$} \\
\hline 2\% Biomass R2 & $091.12^{\mathrm{c}}+5.67$ & $0.97^{b} \pm .02$ & 187.44 & $2.06^{\mathrm{c}}+.16$ & $1.95^{\mathrm{a}} \pm .05$ & $15.47^{\mathrm{a}} \pm 1.1$ & $30.87^{\mathrm{a}} \pm 1.21$ \\
\hline $4 \%$ Biomass R4 & $111.16^{\mathrm{a}+5.63}$ & $1.07^{\mathrm{a}}+.03$ & 421.16 & $3.80^{\mathrm{b}}+.14$ & $1.06^{\mathrm{b}}+.04$ & $08.51^{\mathrm{b}+1.2}$ & $16.80^{\mathrm{b}+1.19}$ \\
\hline $6 \%$ Biomass R6 & $106.98^{\mathrm{b}}+4.62$ & $1.05^{\mathrm{a}}+.02$ & 617.98 & $5.97^{\mathrm{a}} \pm .20$ & $0.70^{c} \pm .04$ & $05.62^{c^{-}+1.2}$ & $11.09^{\mathrm{c}}+2.23$ \\
\hline \multicolumn{8}{|l|}{ Feedings/d (F) } \\
\hline 2 meals/d, F2 & $103.10^{\mathrm{a}} \pm 5.63$ & $1.03^{\mathrm{a}} \pm .04$ & 408.83 & $3.88^{\mathrm{a}} \pm .19$ & $1.23^{\mathrm{a}} \pm .03$ & $09.80^{\mathrm{a}} \pm 1.1$ & $19.47^{\mathrm{a}} \pm 1.41$ \\
\hline 3 meals/d, F3 & $103.07^{\mathrm{a}} \pm 4.70$ & $1.03^{\mathrm{a}} \pm .03$ & 408.87 & $3.88^{\mathrm{a}} \pm .21$ & $1.23^{\mathrm{a}+.05}$ & $09.94^{\mathrm{a}} \pm 1.2$ & $19.71^{\mathrm{a}} \pm 1.95$ \\
\hline \multicolumn{8}{|l|}{ Stocking density (D) } \\
\hline $25 \mathrm{fish} / \mathrm{m}^{3} \mathrm{D} 25$ & $108.13^{\mathrm{a}} \pm 5.59$ & $1.05^{\mathrm{a}} \pm .02$ & 418.02 & $3.71^{\mathrm{b}} \pm .15$ & $1.27^{\mathrm{a}} \pm .04$ & $10.16^{\mathrm{a}} \pm 1.2$ & $20.25^{\mathrm{a}} \pm 1.32$ \\
\hline $50 \mathrm{fish} / \mathrm{m}^{3} \mathrm{D} 50$ & $098.04^{\mathrm{b}}+3.91$ & $1.00^{\mathrm{b}} \pm .04$ & 399.69 & $3.99^{\mathrm{a}}+.18$ & $1.20^{\mathrm{a}} \pm .03$ & $09.58^{\mathrm{b}} \pm 1.3$ & $18.92^{\mathrm{b}}+1.97$ \\
\hline \multicolumn{8}{|l|}{$\begin{array}{lllll}\boldsymbol{R} & \mathbf{X} & \boldsymbol{F} & \mathbf{X} & \boldsymbol{D}\end{array}$} \\
\hline R2 $\times$ F2 $\times$ D25 & $093.02^{\mathrm{d}} \pm 6.38$ & $0.98^{\mathrm{d}} \pm .05$ & 187.90 & $2.02^{\mathrm{C}}+.11$ & $1.98^{\mathrm{a}} \pm .12$ & $15.66^{\mathrm{a}} \pm 1.2$ & $31.46^{\mathrm{a}} \pm 2.01$ \\
\hline $\mathrm{R} 2 \times \mathrm{F} 2 \times \mathrm{D} 50$ & $086.66^{\mathrm{d}}+5.46$ & $0.94^{\mathrm{d}_{ \pm}^{-}}-04$ & 183.97 & $2.21^{\mathrm{c}} \pm .13$ & $1.89^{\mathrm{a}}+.09$ & $14.90^{\mathrm{a}} \pm 1.3$ & $29.73^{\mathrm{a}} \pm 1.51$ \\
\hline $\mathrm{R} 2 \times \mathrm{F} 3 \times \mathrm{D} 25$ & $096.48^{\mathrm{cd}^{-}}+4.72$ & $1.00^{\mathrm{d}} \pm .04$ & 191.95 & $1.99^{\mathrm{c}} \pm .15$ & $2.01^{\mathrm{a}} \pm .10$ & $16.19^{\mathrm{a}} \pm 1.4$ & $32.19^{\mathrm{a} \pm 1.49}$ \\
\hline $\mathrm{R} 2 \times \mathrm{F} 3 \times \mathrm{D} 50$ & $088.33^{\mathrm{d}} \pm 4.83$ & $0.95^{\mathrm{d}} \pm .03$ & 185.92 & $2.10^{\mathrm{C}} \pm .17$ & $1.90^{\mathrm{a}} \pm .14$ & $15.11^{\mathrm{a} \pm 1.3}$ & $30.11^{\mathrm{a}} \pm 1.95$ \\
\hline $\mathrm{R} 4 \times \mathrm{F} 2 \times \mathrm{D} 25$ & $119.39^{\mathrm{a}} \pm 5.72$ & $1.11^{\mathrm{a}+.03}$ & 437.54 & $3.66^{\mathrm{b}} \pm .14$ & $1.09^{\mathrm{b}} \pm .13$ & $08.82^{\mathrm{b}} \pm 1.4$ & $17.63^{\mathrm{b}} \pm 1.73$ \\
\hline $\mathrm{R} 4 \times \mathrm{F} 2 \times \mathrm{D} 50$ & $107.37^{\mathrm{bc}}+4.58$ & $1.05^{\mathrm{bc}} \pm .04$ & 414.02 & $3.86^{\mathrm{b}}+.13$ & $1.04^{\mathrm{b}}+.14$ & $08.21^{\mathrm{b}} \pm 1.3$ & $16.04^{\mathrm{b}}+2.19$ \\
\hline $\mathrm{R} 4 \times \mathrm{F} 3 \times \mathrm{D} 25$ & $115.30^{\mathrm{a}} \pm 5.38$ & $1.09^{\mathrm{a}} \pm .03$ & 427.91 & $3.71^{\mathrm{b}} \pm .11$ & $1.08^{\mathrm{b}} \pm .12$ & $08.67^{\mathrm{b}} \pm 1.3$ & $17.07^{\mathrm{b}} \pm 1.21$ \\
\hline $\mathrm{R} 4 \times \mathrm{F} 3 \times \mathrm{D} 50$ & $102.56^{\mathrm{bc}}+5.70$ & $1.03^{\mathrm{bc}}+.02$ & 405.15 & $3.95^{\mathrm{b}}+.16$ & $1.01^{\mathrm{b}}+.11$ & $08.35^{\mathrm{b}} \pm 1.0$ & $16.46^{\mathrm{b}+1.60}$ \\
\hline R6 $\times$ F2 $\times$ D25 & $113.29^{\mathrm{a}}+3.38$ & $1.08^{\mathrm{a}} \pm .05$ & 634.86 & $5.60^{\mathrm{a}} \pm .14$ & $0.72^{\mathrm{c}} \pm .11$ & $05.83^{\mathrm{c}} \pm 1.1$ & $11.55^{\mathrm{c}} \pm 1.45$ \\
\hline R6 $\times$ F2 $\times$ D50 & $098.85^{\mathrm{cd}}+4.46$ & $1.01^{\mathrm{cd}}+.03$ & 594.71 & $6.02^{\mathrm{a}}+.13$ & $0.67^{c}+.18$ & $05.36^{\mathrm{C}}+1.0$ & $10.39^{\mathrm{c}}+1.81$ \\
\hline R6 $x$ F3 $\times$ D25 & $111.32^{\mathrm{ab}} \pm 4.12$ & $1.07^{\mathrm{ab} \pm .04}$ & 627.94 & $5.64^{\mathrm{a}} \pm .15$ & $0.71^{\mathrm{c}} \pm .19$ & $05.78^{\mathrm{C}} \pm 1.2$ & $11.62^{\mathrm{C}} \pm 2.32$ \\
\hline R6 $\times$ F3 $\times$ D50 & $104.45^{\mathrm{bc}} \pm 4.35$ & $1.04^{\mathrm{bc}} \pm .05$ & 614.37 & $5.88^{\mathrm{a}} \pm .18$ & $0.68^{\mathrm{c}} \pm .30$ & $05.52^{\mathrm{C}} \pm 1.2$ & $10.81^{\mathrm{c}} \pm 1.54$ \\
\hline
\end{tabular}

a,b,c and d values in the same row with different superscript letters are significantly different $(P<0.05)$.

In Trial 2 results concerning the effect of using a single diet (25\% CP) with different feeding schedules on fish composition (Table 5) showed that increasing either feeding rate form 2 to $6 \%$ of biomass (F) or feeding frequencies from 2 to 3 meals per day and stocking density from 25 to 50 fish $/ \mathrm{m}^{3}$ had no significant effect on fish body proximate composition 
Table 5. Proximate composition (\%DM) of hybrid tilapia muscles fed on test diets in trial 2 (mean \pm SE).

\begin{tabular}{|c|c|c|c|c|c|}
\hline Treatment & Moisture & $\begin{array}{c}\text { Crude } \\
\text { protein }\end{array}$ & $\begin{array}{l}\text { Crude } \\
\text { lipid }\end{array}$ & Ash & $\begin{array}{c}\text { Gross energy* } \\
\left(\mathrm{KJg}^{-1}\right)\end{array}$ \\
\hline Initial & $75.61+1.12$ & $15.91+0.94$ & $4.15+0.42$ & $4.33+0.35$ & $5.45+0.15$ \\
\hline \multicolumn{6}{|l|}{ Feed ration $(R)$} \\
\hline $2 \%$ Biomass R2 & $76.51+0.85^{\mathrm{a}}$ & $15.87 \pm 0.46^{\mathrm{a}}$ & $4.10 \pm 0.21^{\mathrm{b}}$ & $3.53 \pm 0.46^{\mathrm{b}}$ & $5.42^{\mathrm{a}}+0.13$ \\
\hline 4\% Biomass R4 & $76.21+0.87^{\mathrm{a}}$ & $15.93 \pm 0.35^{\mathrm{a}}$ & $4.24 \pm 0.32^{\mathrm{a}}$ & $3.62 \pm 0.35^{\mathrm{a}}$ & $5.49^{\mathrm{a}} \pm 0.18$ \\
\hline $6 \%$ Biomass R6 & $76.18+0.77^{\mathrm{a}}$ & $15.97 \pm 0.29^{\mathrm{a}}$ & $4.28+0.22^{\mathrm{a}}$ & $3.57 \pm 0.47^{\mathrm{a}}$ & $5.51^{\mathrm{a}}+0.15$ \\
\hline \multicolumn{6}{|l|}{ Feedings/d (F) } \\
\hline 2 meals/d, F2 & $76.40 \pm 0.84^{\mathrm{a}}$ & $15.86 \pm 0.66^{\mathrm{a}}$ & $4.19 \pm 0.43^{\mathrm{a}}$ & $3.55 \pm 0.55^{\mathrm{a}}$ & $5.45^{\mathrm{a}} \pm 0.18$ \\
\hline 3 meals/d, F3 & $76.21+0.82^{\mathrm{a}}$ & $15.98+0.73^{\mathrm{a}}$ & $4.23 \pm 0.51^{\mathrm{a}}$ & $3.60 \pm 0.51^{\mathrm{a}}$ & $5.49^{\mathrm{a}} \pm 0.15$ \\
\hline \multicolumn{6}{|l|}{ Stocking density (D) } \\
\hline $25 \mathrm{fish} / \mathrm{m}^{3} \mathrm{D} 25$ & $76.27 \pm 0.93^{\mathrm{a}}$ & $16.02 \pm 0.85^{\mathrm{a}}$ & $4.17 \pm 0.29^{\mathrm{a}}$ & $3.55 \pm 0.45^{\mathrm{a}}$ & $5.48^{\mathrm{a}} \pm 0.16$ \\
\hline $50 \mathrm{fish} / \mathrm{m}^{3} \mathrm{D} 50$ & $76.33+0.75^{a}$ & $15.83+0.59^{\mathrm{a}}$ & $4.24+0.56^{\mathrm{a}}$ & $3.60 \pm 0.39^{\mathrm{a}}$ & $5.46^{\mathrm{a}}+0.13$ \\
\hline \multicolumn{6}{|l|}{$\begin{array}{lllll}R & \mathbf{X} & \boldsymbol{F} & \mathbf{X} & \boldsymbol{D} \\
\end{array}$} \\
\hline $\mathrm{R} 2 \times \mathrm{F} 2 \times \mathrm{D} 25$ & $76.52+0.65^{\mathrm{a}}$ & $15.87 \pm 0.63^{\mathrm{ab}}$ & $4.04+0.42^{b}$ & $3.57+0.29^{\mathrm{bc}}$ & $5.39^{b}+0.17$ \\
\hline $\mathrm{R} 2 \times \mathrm{F} 2 \times \mathrm{D} 50$ & $76.59+0.73^{\mathrm{a}}$ & $15.79 \pm 0.54^{\mathrm{ab}}$ & $4.09+0.47^{\mathrm{b}}$ & $3.53+0.43^{\mathrm{bc}}$ & $5.39^{\mathrm{b}}+0.15$ \\
\hline R2 $x \quad F 3 \times$ D25 & $76.47+0.83^{\mathrm{a}}$ & $15.96+0.65^{\mathrm{a}}$ & $4.17 \pm 0.35^{\mathrm{ab}}$ & $3.40+0.52^{\mathrm{c}}$ & $5.47^{\mathrm{ab}}+0.15$ \\
\hline R2 $x \quad F 3 \times$ D50 & $76.45+0.66^{\mathrm{a}}$ & $15.84+0.73^{\mathrm{ab}}$ & $4.11+0.39^{b}$ & $3.60+0.43^{\mathrm{ab}}$ & $5.41^{\mathrm{b}}+0.17$ \\
\hline $\mathrm{R} 4 \times \mathrm{F} 2 \times \mathrm{D} 25$ & $76.29+0.75^{\mathrm{a}}$ & $16.08+0.81^{\mathrm{a}}$ & $4.13+0.42^{\mathrm{b}}$ & $3.50 \pm 0.36^{\mathrm{bc}}$ & $5.48^{\mathrm{ab}}+0.14$ \\
\hline $\mathrm{R} 4 \times \mathrm{F} 2 \times \mathrm{D} 50$ & $76.39+0.83^{\mathrm{a}}$ & $15.65+0.34^{\mathrm{b}}$ & $4.27+0.52^{\mathrm{a}}$ & $3.69+0.50^{\mathrm{a}}$ & $5.43^{b}+0.18$ \\
\hline $\mathrm{R} 4 \times \mathrm{F} 3 \times \mathrm{D} 25$ & $76.37+0.93^{\mathrm{a}}$ & $15.83+0.56^{\mathrm{ab}}$ & $4.24+0.43^{\mathrm{ab}}$ & $3.56 \pm 0.21^{\mathrm{bc}}$ & $4.46^{\mathrm{ab}} \pm 0.13$ \\
\hline $\mathrm{R} 4 \times \mathrm{F} 3 \times \mathrm{D} 50$ & $75.80 \pm 0.85^{\mathrm{a}}$ & $16.15 \pm 0.78^{\mathrm{a}}$ & $4.31 \pm 0.49^{\mathrm{a}}$ & $3.74 \pm 0.34^{\mathrm{a}}$ & $5.57^{\mathrm{a}}+0.14$ \\
\hline R6 $\times$ F2 $\times$ D25 & $76.14 \pm 0.76^{\mathrm{a}}$ & $16.10 \pm 0.65^{\mathrm{a}}$ & $4.23 \pm 0.37^{\mathrm{ab}}$ & $3.53 \pm 0.51^{\mathrm{bc}}$ & $5.52^{\mathrm{a}} \pm 0.18$ \\
\hline $\mathrm{R} 6 \times \mathrm{F} 2 \times \mathrm{D} 50$ & $76.48 \pm 0.93^{\mathrm{a}}$ & $15.67 \pm 0.47^{\mathrm{b}}$ & $4.36 \pm 0.58^{\mathrm{a}}$ & $3.49 \pm 0.33^{\mathrm{c}}$ & $5.47^{\mathrm{ab}} \pm 0.19$ \\
\hline R6 $\times$ F3 $\times$ D25 & $75.81 \pm 0.84^{\mathrm{a}}$ & $16.25 \pm 0.83^{\mathrm{a}}$ & $4.22 \pm 0.36^{\mathrm{ab}}$ & $3.72 \pm 0.37^{\mathrm{a}}$ & $5.56^{\mathrm{a}} \pm 0.16$ \\
\hline R6 $x$ F3 $x$ D50 & $76.27 \pm 0.85^{\mathrm{a}}$ & $15.87 \pm 0.92^{\mathrm{ab}}$ & $4.31 \pm 0.47^{\mathrm{a}}$ & $3.55 \pm 0.42^{\mathrm{bc}}$ & $5.50^{\mathrm{a}}+0.18$ \\
\hline
\end{tabular}

a,b and c values in the same row with different superscript letters are significantly different $(P<0.05)$.

* Muscle gross energy (K cal/100g muscle) by NKc (1983) $=5.7$ x \% crude protein $+9.5 \mathrm{x} \%$ crude lipid. $(\mathrm{Kj}=4.186 \mathrm{~K} \mathrm{cal})$

\section{DISCUSSION}

To obtain a good hybrid tilapia production, it is necessary to improve feed quality, especially protein and energy contents. Practical feeds for growout tilapias usually contain 25-35\% CP (Swick, 2001). Similarly, in the present work, a 25\% CP diet gave the best growth, feed utilization and body composition for monosex hybrid tilapia, with a starting weight around $30 \mathrm{~g}$ (Tables 2\&3). These findings are in agreement with earlier studies on both Nile and blue tilapias (Wassef, 2005) and for their hybrid (Twibell \& Brown, 1998; Wang et al.,2005).

Furthermore, the $\mathrm{P} / \mathrm{E}$ ratio of (146.85 $\mathrm{mg} \mathrm{CP} / \mathrm{kj} \mathrm{GE}$ ) is recommended for best growth and feed performance of hybrid tilapia (O. niloticus $X$ O. aureus) (Table 2). The previous study of Santiago \& Laron (1991) estimated: $104 \& 111$ mg P/kj ME (106 mg P/kcal DE for 7.5g O. aureus (Winfree \& Stickney, 1981). However, a relatively higher P/E ratio (>230 kcal ME/100g diet in 24\% CP) was required for hybrid tilapia (O. niloticus $X$ O. aureus), reared in seawater (Shiau \& Huang, 1990). Improper balance of P/E ratios will lead to poor growth; however, this growth reduction was not as pronounced as that of diets with excessive P/E ratios (Santiago \& Laron, 1991). Shiau \& Lan (1996) and 
Samantaray \& Mohanty (1997) suggested that any inadequate dietary P/E ratio will result in lower growth as well as lower protein and energy utilization. These variations may be due to the following two aspects besides fish species; first is experimental dietary $\mathrm{P} / \mathrm{E}$ level. Thus, the different designs of dietary nutrient level affect the estimation of nutrient requirement (Mercer, 1982). The other is the difference in dietary ingredients readily digestible and provide sufficient essential amino acids, (NRC, 1993). Therefore, the optimal dietary P/E ratio varied for the same physiological fuel values (Catacutan and Coloso, 1995).

Several authors recommended the restricted feeding regime for various tilapia species and indicated that adlibitum feeding may result in feed waste (Sumagaysay, 1998). For hybrid tilapia (O. niloticus X O. aureus) results of the present study suggested a 25\% CP diet provided at $4 \%$ of biomass, agreement with results obtained by De Silva et al., (1989) for Nile tilapia. Furthermore, increasing feeding level of $O$. niloticus may have an increased rate of feed passage through the digestive tract, leading to depressed digestibility of gross energy (Meyer-Burgdorff et al., 1989). The rate at which food is consumed and the efficiency with which it is utilized are prime factors in determining growth rate. More recently, gastric evacuation rate and return of appetite following a satiation meal was estimated for $O$. niloticus to be approximately $4 \mathrm{~h}$ (Riche et al., 2004).

Concerning the feeding rate, lipid content of flesh changed significantly $(\mathrm{P}<0.05)$ in the current work (Table 5). This situation has already been demonstrated with yellow tail flounder (Limanda ferruginea) juveniles reported by Puvanendran et al. (2003) and with sea bass (Dicentrachus labrax) by Eroldogan et al. (2004).

The results presented in Table (4) showed that increasing the feeding frequencies from two to three meals daily did not increase weight gain. NRC (1993) reported a positive relationship between growth and feeding frequency in Nile tilapia, that might be due to the fact that fish appeared to be continuous feeder in its behavior with smaller stomach capacity. However, fish fed at lower frequencies, had less opportunity to feed during the day and were therefore more active during feeding and developed food anticipatory activity, prior to feeding which benefits the fish by optimizing feed intake and feeding efficiency (Chen \& Purser, 2001).

The growth performance and feed conversion ratio of hybrid tilapia were significantly $(\mathrm{P}<0.05)$ affected by the tested densities of 25 or $50 \mathrm{fish} / \mathrm{m}^{3}$ in the present study(Table 4). The inverse relation between density and both fish growth and feed efficiency in venires species have been shown (Maragoudaki et al., 1999), due to competition (Helser \& Almedia, 1997), space limitation (Ewing et al., 1998), insufficient food and low dissolved oxygen (Yi et al., 1996). Vijayan \& Leatherland (1988) suggested that the pituitary-thyroid axis may be a possible route for growth inhibition at high stocking density. Though in earlier studies of rearing Nile and blue tilapia (O. niloticus \& O. aureus) or 
their hybrid, a stocking density of $25 \mathrm{fish} / \mathrm{m}^{3}$ was commonly adopted in cages (Wassef et al., 2003), in fiberglass tanks (Abdel-Hakim et al., 2003) .

It is evident that some interaction between feeding level and the frequency of feeding occurs in terms of energy requirements for maintenance and in terms of protein contribution to such needs (Kaushik \& Gomes, 1988). Though, difference in experimental conditions (feeding methods and stocking density), size of fish, and intensive culture systems may effect the requirements of dietary composition and culture management (Webster et al., 1992).

\section{Conclusions}

Based on the obtained results it is recommended to use a diet containing $25 \% \mathrm{CP}+16.74 \mathrm{~kg}$ g-1 at a feeding rate of $4 \%$ of fish biomass when fish stocked at 25 fish $/ \mathrm{m} 3$ for best growth and nutrient utilization of Nile tilapia. The diet should be fed twice daily.

\section{REFERENCES}

Abdel-Hakim N.F.; Al-Azab A.A. and El-Kholy K.F. (2003). Effect of feeding some full-fat oil seeds on performance of hybrid tilapia (Oreochromis niloticus $X$ O.aureus) reared in tanks. Egypt. Journal of Nutrition Egyptian and Feeds, 6: 389-403.

Ai Q.; Mai K.; Li H.; Zhang C.; Zhang L.; Duan Q.; Tan B.; Xu W.; Ma H.; Zhange W. and Liufu, Z. (2004). Effects of dietary protein to energy ratios on growth and body composition of juvenile Japanese sea bass, Lateolabrax japonicus. Aquacult., 230, 507-516.

AOAC, (1995). Official Methods of Analysis.(Association of Official Analytical Chemists). $16^{\text {th }}$ ed. AOAC, Arlington, VA, USA, $1298 \mathrm{pp}$.

APHA, (1995). American Public Health Association. Standard methods for the examination of water and waste water. $18^{\text {th }}$ ed. Washington, DC. USA, $769 \mathrm{pp}$.

Baskerville - Bridges B. and Kling L.J. (2002). Larval culture of Atlantic cod (Gadus morhua) at high stocking densities. Aquacult., 181: 61-69.

Bligh E.G. and Dyer F.G. (1959). A rapid methods of total lipid excretion and purification. Candian Journal of Biochemistry and Physiology, 37: 911-917. 
Catacutan M. R. and Coloso R. M. (1995). Effect of dietary protein to energy ratio in growth , survival , and body composition of juvenile Asian sea pass, Lates calcarifer, quacult.,131: 125-133.

Chen W. M. and Purser G. J. (2001). The effect of feeding regime on growth, locomotors activity pattern and the development of food anticipatory activity in greenback flounder. J.of Fish Bio., 58: 177-187.

De Silva, S. S.; Gunasekera, R. M. and Atapattu, D. (1989). The dietary protein requirements of young tilapia and an evaluation of the least cost dietary protein level. Aquacult., 80: 271-284.

El-Sayed, A. M. (2006). Tilapia Culture. CABI Publ. Wilingford, Oxon, U.K. 304 pp.

Eroldogan, O.T.; Kumlu, M. and Aktas, M. (2004). Optimum feeding rates for European sea bass Dicentrarchus labrax L. reared in sea water and freshwater. Aquacult., 31: 501-515.

Ewing, R.D.; Sheahan, J.E.; Lewis, M.A. and Plamisano, A.N. (1998). Effects of rearing density and race way conformation on growth, food conversion, and survival of Juvenile spring chinook salmon. Progressive Fish Culture, 60: 167-178.

Helser, T.E. and Almeida, F.P. (1997). Density dependent growth and sexual maturity of silver hake in the northwest Atlantic. Journal of Fish Biology, 51: 607-623.

Kaushik, S.J. and Gomes, E.F. (1988). Effect of frequency of feeding on nitrogen and energy balance in rainbow trout under maintenance conditions. Aquacult., 73: 207-2216.

Maragoudaki, D.; Paspatis, M. and Kentouri, M. (1999). Influence of stocking density on growth of juvenile red progy (Pagrus pagrus L.) under different feeding conditions. Aquacult. Research, 30: 501-508.

Meyer-Burgdorff, K.H.; Osman, M.F. and Gunther, K.D. (1989). Energy metabolism in Oreochromis niloticus. Aquacult., 79: 283

NRC (1983). Nutrition requirement of Worm Water Fishes \& Shell Fishies. National Academy of scie. Washington, DC.

NRC (1993). Nutrient Requirements of Fish. National Academy press, Washington, DC. 
Puvanendran, V.; Boyce, D.L. and Brown J.A. (2003). Food ration requirements of $\mathrm{O}^{+}$yellowtail flounder Limanda ferruginea (Storer) Juveniles. Aquacult., 220: 459-475.

Riche, M.; Haley, D.I.; Oetker, M.; Garbrecht, S. and Garling, D.L. (2004). Effect of feeding frequency on gastric evacuation and the return of appetite in tilapia Oreochromis niloticus (L.). Aquacult., 234: 657673.

Samantaray, K. and Mohanty, S.S. (1997). Interactions of dietary snakehead, Channa striata, Aquacult.,156: 241-249.

Santiago, C.B. and Laron, M.A. (1991). Growth response and carcass composition of red tilapia fed diets with varying protein levels and protein to energy ratios. Proc. $4^{\text {th }}$ Asian Fish Nut. Workshop, 5: 55-62.

Santiago, C.B. and Reyes, O.S. (1993). Effect of dietary lipid sources on reproductive performance and tissue lipid levels of Nile tilapia (Oreochromis niloticus) broodstock. J. of Appied Ichthyol., 9 (1): 33-40.

SAS (Statistical Analysis System Institute), (1996). A Guide to Statistical and Data Analysis Using JMP ${ }^{\circledR}$ and JMP $1 \mathrm{~N} \circledast$ Software. SAS Institute, North Carolina, USA.

Shiau, S.Y. and Huang, S.L. (1990). Influence of varying energy levels with two protein concentrations in diets for hybrid tilapias (Oreochromis niloticus $X$ O.aureus) reared in seawater. Aquacult., 91: 143-152.

Shiau, S.Y. and Lan, C.W. (1996). Optimum dietary protein level and protein to energy ratio for growth of grouper (Epinephelus malabaricus). Aquacult., 145: 259-266.

Sumagaysay, N.S. (1998). Milk fish (Chanos chanos) production and water quality in brackish water ponds at different feeding levels and frequencies. $J$. of Applied Ichthyol., 14(1-2): 81-95.

Swick R.A. (2001). Feed based tilapia culture. ASA Techn. Bull., 49-10 pp.

Twibell, R.G. and Brown, B.P. (1998). Optimal dietary protien concentration for hybrid tilapia (Oreochromis niloticus $\times O$. aureus ) fed all - plant diet. J. The world aquacult. Soci., 29 (1):9-10. 
Vijyan, M.M. and Leatherland, J.F. (1988), Effect of stocking density on the growth and stress-response in Brook Charr, Salvelinus fontinalis. Aquacult., 75:159-170.

Wang, Y.; Liu, Y.; Tian, L.; Du, Z.; Wang, J.; Wang, S. and Xiao, W.(2005). Effect of dietary carbohydrates level on growth and body composition of juvenile tilapia , (Oreochromis niloticus x O. aureus). Aquacult., Rese.., 36:1408 abs.

Wassef, E.A. (2005). Alternative protein sources for fish feeds in Egypt. Cahier Options Meditr. (63) Mediterranean Fish Nutrition: pp.127-141

Wassef, E.A.; Shalaby, S.H.; Mabrouk, H.A. and Sakr, E.M. (2003). Experiments on farming fish into ground water. Egyptian $J$. of Nut. \& Feeds, 6: 371-388.

Webster, C.D.; Tidwell, J.H. and Yancey, D.H. (1992). Effect of protein level and feeding frequency on growth and body composition of (age-reared channel catfish. The Progressive Fish Culturist, 54: 92-96.

Winfree, R.A. and Stickney, R.R. (1981). Effects of dietary protein and energy on growth, feed conversion efficiency and body composition of Tilapia aurea. J. of Nut.111:1001-1012.

Woods, C.M.C. (2003). Effect of stocking density and gender segregation in the seahorse, Hippocampus abdominalis. Aquacult., 218-167pp.

Yi, Y.; Lin, C.K. and Diana, J.S. (1996). Influence of Nile Tilapia (Oreochromis niloticus) stocking density in cages on their growth and yield in cages and in ponds containing the cages. Aquacult., 146:205-215. 